\title{
基于 GPS 网络 RTK 定位在土地勘探测量中的应用
}

\section{Application of RTK Positioning Based on GPS Network in Land Exploration Survey \\ 徐楚}

$\mathrm{Chu} \mathrm{Xu}$

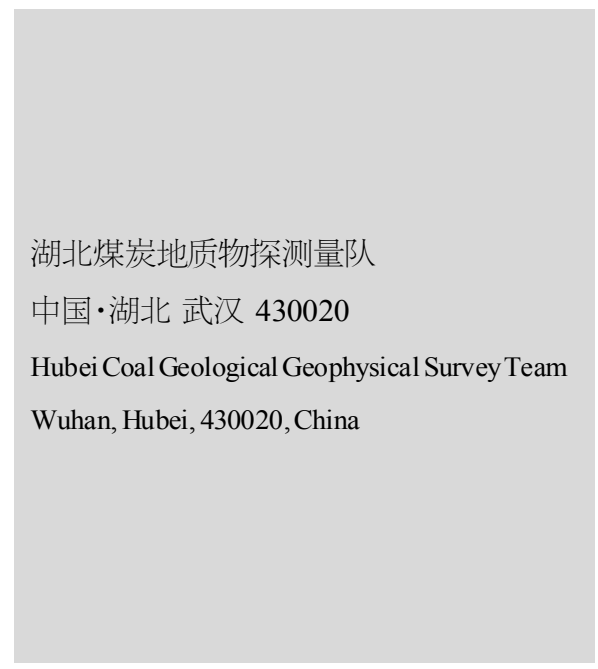

【摘要】随着社会的不断发展, GPS 技术应用范围也逐渐扩大。在进行土地勘测工作中, 通过 GPS 网络 RTK 定位系统可以明确地对土地情况进行测量, 该技术具有精准性高、自 动化以及高效益的特点, 受到科技工作者的广泛认可。论文针对 GPS 定位系统在土地勘测 工作的实际应用展开分析工作。

【Abstract】With the continuous development of society, the application of GPS technology has gradually expanded. In the process of land survey, the RTK positioning system based on GPS network can measure the land situation clearly. This technology has the characteristics of high accuracy, automation and high efficiency, and is widely recognized by scientific and technological workers. This paper analyzes the practical application of GPS positioning system in land survey.

【关键词】GPS 网络; RTK 技术; 土地勘测; 实际应用

【Keywords】GPS network; RTK technology; land survey; practical application 【DOI】10.36012/se.v1i2.906

\section{GPS-RTK 技术介绍}

\section{1 概念}

从整体角度来看, GPS-RTK 定位系统可以划分为 4 部 分: 基准站、移动站、数据链以及控制软件。基准站主要在 GPS 接收机的基础上进行架设。移动站并不是独立作业的,需要与 其他环节联合作业, 如中杆、电子手薄等, 联合作业可以使数 据收集更为便捷,也可以开展坐标放样。数据链主要用于联 系移动站与基准站。由基准站将数据传输, 移动站进行数据 接收 ${ }^{[1]}$ 。过程中需要利用移动设备作为传输通道, 使用设备的 大小和基准站与移动站之间的距离有关, 并且二者周边环境 也会对数据传输效率产生影响。控制软件主要负责处理数据, 最主要的功能就是计算流动站的数据, 为使用 GPS-RTK 技 术时提供数据依据。

\section{2 工作原理}

GPS-RTK 技术主要由基准站与流动站两部分组成, GPS 接收器安装在基准站上, 主要负责对所有的 GPS 卫星开展不 间断的探测, 并将所探测的数据通过无线电的方式进行传输,
用户的观测站进行信号接收。用户站具有实时流动的特点, 在 此基础上接收 GPS 卫星所传输的信号, 同时, 利用无线电装 备对观测数据进行接收，将格局相对定位的原理作为分析依 据,通过精密的计算得出用户站的三维坐标。GPS-RTK 定位 技术精密性较高, 采取差分定位的方式可以有效地减小误差, 在一定程度上可以消除误差,进一步实现精确定位。

\section{3 应用的注意事项}

在应用 GPS-RTK 技术进行土地勘测工作过程中，需要 注意一些事项: 第一点,在基站选择方面尽可能选择视野较为 开阔的位置, 上方及周围不可以存在成片的障碍物, 影响信号 传输, 进而保证 GPS 卫星的连续观测与传输信号的质量。第 二点, 为了避免外在因素对 GPS 卫星信号的影响, 需要保障 基站周围 $200 \mathrm{~m}$ 范围内不能有高强度电磁波干扰源，其发射 的电磁波对信号质量存在较大的影响。比如, 高压输电线、大 功率无线电发射设施等。第三点, 为了避免多路径效应对定位 数据的影响, 检测站位置尽量远离电磁波信号较为强烈的地 方, 比如, 成片水域、高层建筑等 ${ }^{[2]}$ 。第四点, 为后续工作提供 便利, 观测站位置应该选择交通发达、上点较为容易的地方。 
测绘技术 Surveying and Mapping Technique

\section{4 局限性}

在土地勘测工作中，采用 GPS-RTK 技术存在一定的局 限性, 主要表现为几方面: (1)使用者在本地架设观测站, GPS 定位数据的误差随着距离增长而发生改变, 误差增长会导致 参考站与流动站的距离受到制约, 不得超过 $15 \mathrm{~km}$, 并且其可 行性与可靠性也随距离而降低; 2RTK 自身不具备几何检核 条件, 所以在假设加密控制点之前, 需要强化检核, 在进行代 替一二级点时, 需要在不同的基准站上进行测验, 结果取中 值, 可以有效地减小误差; (3RTK 定位数据的处理方式主要 是依靠流动站与基准站之间的单基线，由于无线电信号的传 输质量与观测数据质量对定位精度的影响非常大, 所以, 应该 选择测量区域内制高点出进行架构, 从而提高观测天线高度; (4)架构参考站时需要注意周边环境, 原理通讯塔、微波塔等大 型电磁波发射源，尽量保持距离为 $200 \mathrm{~m}$ 之外。对于通信线 路、高压输电线路应保持 $50 \mathrm{~m}$ 距离以上 ${ }^{[3]}$ 。

\section{GPS-RTK 技术在地勘探测量中的作 业流程}

\section{1 测区踏勘}

在 GPS 外部测量工作时, 应做好准备工作, 首先对观测 区域周边环境进行勘察, 通过对观测的真实情况进行了解, 选 择更为合理的位置进行基站架设，为了避免外在因素影响传 输信号的质量, 架设基站的位置尽可能在观测区域的中心, 选 择地势较高、上方没有遮挡物并且基站可以进行全面覆盖的 地方。基站附近 $200 \mathrm{~m}$ 处尽量避免出现高压线、无线电发射台 以及电视转播台等干扰源, 以至于造成数据链丢失以及出现 多路径效应的问题,确保在基站附近没有 GPS 信号反射源。

\section{2 计算测区转换参数}

在实际工作中, GPS 定位系统所提供的 WGS84 大地坐标 并没有太多的实际意义,许多工程并不重视, 在观测过程中需 要将 84 坐标转变为工程施工坐标或者国家平面坐标。可以选 取高斯投影的方式实现 WGS84 到国家平面坐标的转化, 在一 般情况下,通常选取控制点成果求取“区域性”的地方转化参 数,需要具备前提条件:第一点, 足够多的控制点数量。通常来 说, 至少需要 3 个平面控制, 根据观测地域的地形地貌进行高 程控制, 控制点数量会增多, 进而保障拟合精准度的要求。第 二点,把握控制点的分布合理性与控制范围。通常情况下, 控 制点的控制范围需要保障可以覆盖整个观测区域, 并且邻近 的两个控制点相距应该在 $3 \sim 5 \mathrm{~km}$ ，分布的合理性就是保障控 制点均匀分布，如果条件允许，控制点越多效果越好。第三 点,保障每个控制点之间的位置关系的精确度, 比如, 地方坐
标 XYZ 和大地坐标 BLH, 进而实现参数转换

\section{3 确保转换关系的正确性}

在实际测量工作中, 通常会出现几种可能: (1)在较为广阔 的观测地区只存在有限等级控制点的地方坐标 XYZ。所以, 需要结合观测地点的实际情况进行加密的控制测量, 通过 GPS 技术将静态数据整合进行统一网平差, 从而分析出精度 较为准确的地方坐标与 WGS84 坐标。(2)由于观测地区已经存 在较为充足控制点的地方坐标和 WGS84 坐标, 所以, 该区域 的数据较为精准, 并且每一个控制点具有稳定的位置关系。(3) 当观测区域只存在较多控制点的地方坐标，并且控制点相对 位置较为精确, 但是不存在 WGS84 坐标。基于这种情况, 可以 选择 RTK 技术测量方式, 通过基准站作为起算位置, 具体坐 标位置可以由 GPS 卫星技术观测确定, 虽然其精确度有限, 但是对 RTK 观测结果没有太大影响。随后, 通过基准站确定 每个控制点的具体精确位置关系, 同时对 WGS84 大地坐标进 行实时观测。该方式在实际应用中会存在一些问题,如 RTK 的作用距离有限、控制点之间距离过远等。上述 3 点中在实际 操作时, 都必须保障控制点的地方坐标准确, 此外该控制点的 大地坐标也应该准确。通过静态数据平差计算出的大地坐标, 与 RTK 不能混合使用, 由于二者起算基准不同,会导致转化 参数出现偏差。

\section{3 基于 GPS 网络 RTK 定位在土地勘探 测量中的具体应用}

随着国民经济的高效发展, 中国多数地区不断进行开发 建设工作, 土地测绘工作逐渐频繁, 由于项目工程的要求逐渐 增加, 土地勘察质量要求也越来越高。沿用传统的土地勘测方 式难以满足现代社会发展的趋势, 科学技术不断提高, GPSRTK 技术得到进一步完善升级, 结合 GPS-RTK 技术进行土 地勘测工作可以满足当代工程建设的需求, 可以有效地确定 较为精准的平面坐标, 可以从根本上解决土地勘测中控制点 薄弱这一难题。

\section{1 细部测量}

细部点与界址点通过 RTK 技术或者全站仪进行野外实 时观测平面坐标。开展工作之前, 需要明确基准站的具体精确 坐标, 具体位置可以通过 GPS 静态相对定位技术进行长时间 的观测确定。在使用网络 RTK 技术时, 可以先通过图跟控制 点进行比对校准, 如果校准结果满足限差要求, 才可以开展后 续测量工作。进行观测界址点时, 可以通过全站仪进行极坐标 处理法或者解析交会等方式,观测站架设完成后,需要做好检 查工作, 至少对一个除本站与后视点之外的已知点进行测量 
检查, 进而保障实时观测数据的精准度。GPS-RTK 定位系统 主要由数据通信线路、数据处理中心、基准站等几部分组成。 在观测区域相对制高点架设基准站, 并且在基准站上配备双 频全波长 GPS 接收机, 通过该接收机, 可以保障数据具有高 度精确的双频伪距观测值。在整个测量过程中, 开展地籍控制 测量是整个工作的首要任务, 因为是测绘图件与地籍数据的 基础, 通过控制地籍控制网点的精度以及密度, 主要目的在于 满足该区域测量土地权属范围的特征点, 也就是通常所说的 界址点服务。GPS 地籍网可按测区的范围观测顺序可以划分 为基本网与加密网两类, 由于部分地区的界址点精度与密度 相对较大, 所以, 在满足控制网点的精准度前提下, 需要对控 制点密度逐渐增加进而有利于测定界址点的有效控制, 必要 时可以在 GPS 网络下加密一级图跟导线, 进而可以帮助工作 人员更为直观方便地根据图根点进行界址点测定。在实际情 况下, 需要以全部界址点的解析边长与解析坐标作为参考基 础, 通过 RTK 技术测定地籍数据以及地形要素的几何图形, 以区域草图等相关数据作为基础, 绘制出地籍图。

\section{2 控制测量}

GPS-RTK 定位系统具有良好的操作性, 在进行土地勘测 工作中, 通视、地形以及大气等外在条件对测量结果并没有太 大影响, 控制测量简单便捷, 具有较强的机动性, 相比传统的 测绘方式, 极大地提高了作业效率, 节省了人力物力以及财 力。通过网络 RTK 进行控制测量, 不仅可以满足界址点与地 籍控制测量的精度和密度要求, 并且控制点误差均匀分布, 避 免出现误差积累问题。相比单基站 RKT 技术, 更是克服了随 着观测地区作业半径的不断增加, 控制变量的精度密度与可 靠性逐渐下降的作业难题。在 GPS 地籍控制测量中, 对于控 制点之间一定保证通视不做严格要求, 这样不仅可以有效避 免通过常规的地籍控制点选择存在的局限性, 可以使 GPS 网 络结构对 GPS 网络精度与密度的影响不断减小。在试验区成 功经验的基础上开展实地勘测工作, 首先对该区域的周边环 境进行分析, 随后进行地籍控制测量, 通过选点埋石的方式作 为参考点, 在工作过程中要满足城镇地区地籍测量规范的具 体要求, 在实际勘测前, 需要对观测区域内的已知点进行检 测, 通过仔细检验校对, 保证该地点没有其他问题后, 再开始 进行测量工作。通过这种方式, 可以尽可能地确保观测精度的 可靠性。由于 GPS 技术具备布点灵活、全天候、精度高以及速 度快等一系列优势, 所以 GPS 技术应用逐渐广泛, 在控制测 量工作中都得到充分展现。

\section{3 界址点测量}

开展土地测绘工作最为常见的方式为常规 GPS 结合传
统测量技术, 但是该技术存在一些问题, 比如, 在开展静态控 制测量工作时无法获取控制点的实时坐标, 利用单基站 TRK 技术进行控制测量出现作业距离受到制约。经过调查表明, 通 过 RTK 技术进行整周模糊的的测量，其可靠性最高为 $95 \%$ 。 相比静态 GPS 技术, RTK 存在一些影误差的因素, 比如, 数据 链传输误差等。所以, 相比 GPS 静态测量, 利用 RTK 技术进 行控制测量更加容易出现偏差, 在过程中, 需要进行质量控 制。基于此点, 可以提出相关假设: 基准站与流动站之间的误 差具有很强的关联性。在进行土地勘测工作时, 可以采取 CORS 技术作为参考, 在全球卫星定位系统技术规范与城镇 地籍测量规范等其他技术规范中并没有相关 CORS 技术实际 应用的具体要求, 所以, 本次试验测量可以作为 REK 技术实 际应用的有益尝试。传统的 RTK 会跟随基准站与流动站二者 之间的距离增加而发生改变, 其精度与密度会不断下降, 为了 改善这个问题, 可以采取一些措施。第一点, 减少基准站与用 户之前的距离, 也可以增加基准站网的密度; 第二点, 通过一 些特殊的措施统一传输纠正后的数据, 不仅可以保障其精度 均匀分布, 还能够使基准站与用户之间的距离增加。通过 RTK 技术进行控制测量或者其他测量方式的测绘结果都处 于厘米级, 较差最小值为 $0.1 \mathrm{~cm}$, 最大值为 $1.7 \mathrm{~cm}$, 平均值为 $1.1 \mathrm{~cm}$ 。其中观测点位置中误差为 $0.8 \mathrm{~cm}$, 所以, ZZCORS 技术 中的 RTK 技术完全适用于该地区城镇地籍控制测量。

\section{4 结语}

总而言之, 相比传统土地测绘方式, GPS-RTK 技术具有 较大的优势,一方面在每个测量点之间快捷方便、无需通视, 并且不受地形地势的影响, 极大地提高作业效率; 另一方面, 调整移动站与基准站之间的距离, 使其达到 $10 \mathrm{~km}$ 以上, 不仅 满足了土地测绘的真实需求, 还保障移动站每一控制点的精 准度, 最大程度地避免误差积累, 进而促进土地勘测定界的高 效化、规范化。但是在实际应用中, 采取 GPS-RTK 技术仍然存 在一些问题, 在过程中具有一定的局限性, 比较容易受到外界 遮挡物的影响。随着中国科学技术的不断发展, 网络即时与现 代信息化技术在科研学者的研讨下不断进行升级完善, 到目前 为止, GPS 定位技术应用广泛, 受到世界各地的青睐与关注。

\section{参考文献}

[1]沈翠.刍议 GPSRTK 技术在地质工程测量中的应用 [J].信息系 统工程,2019(5):97.

[2] 王子铭.GPSRTK 技术在地质工程测量中的运用研究与分析 [J].无线互联科技,2019(8):131-132.

[3]杨爱民.GPSRTK 技术在地质工程测量中的应用研究[J].中国 高新科技,2018(2):65-67. 